\title{
Sleeve gastrectomy: an ideal choice for T2DM
}

Michel Gagner

As Ricardo Cohen pointed out (Sleeve gastrectomy: the ideal option for metabolic surgery? Nat. Rev. Endocrinol. 9, doi:10.1038/nrendo.2013.100-c1) $)^{1}$ concerning my News and Views article (Sleeve gastrectomy - the ideal choice for weight-loss surgery. Nat. Rev. Endocrinol. doi:10.1038/ nrendo.2013.100), ${ }^{2}$ the title of my article was on sleeve gastrectomy being the ideal surgery for weight loss, and I did not discuss specifically the subject of diabetes surgery, an entirely different matter. Bariatric surgeons treats type 2 diabetes mellitus (T2DM) in only about one-third of their patients. The meta-analysis of Buchwald et al. on weight loss surgery and also on T2DM, points towards the best operation with resolution of T2DM being the one that gives the most weight loss, hence best results for resolution of T2DM are achieved with biliopancreatic diversions with or without duodenal switch. ${ }^{3,4}$ Mingrone et al. had similarly demonstrated in a randomized study that biliopancreatic diversion gave a much better resolution of T2DM than gastric bypass (95\% versus $75 \%) .{ }^{5}$ Furthermore, the correspondence letter failed to mention that the duodenum bypassing intervention that had a superior effect on T2DM, was in fact a biliopancreatic diversion (BPD), not a gastric bypass. This study was not randomized, and conclusions on exclusion of duodenum not proven, as BPD has a gastrectomy and gastro-ileal anastomosis, which according to the 'hindgut theory' increases early stimulation of L cells (GLP-1 and PYY). ${ }^{6}$ Sleeve gastrectomy, a better choice, is amenable in the future to a duodenal switch (after recurrence of T2DM), and not gastric bypass (a much more complicated proposition) as recurrences and failures of remission of T2DM after gastric bypass are quite well documented $(>40 \%){ }^{7}$

The randomized prospective trial comparing sleeve gastrectomy and gastric bypass by Karamankos et al. has clearly demonstrated a superior weight loss, greater appetite suppression and greater decrease in ghrelin with sleeve gastrectomy. ${ }^{8,9}$ The randomized trial by Lee et al. I also think is flawed, as the sleeve gastrectomy was not performed with a bougie, making a much larger sleeve, that is subjected to a much higher failure rate, and the 'mini' gastric bypass (non Rouxen-Y) studied is a nonstandard operation at the moment. ${ }^{10}$ The trial by Schauer et al. did not demonstrate any significant differences between sleeve and bypass at 1 year. ${ }^{11}$ In this trial, sleeves were performed over a gastroscope, which introduces air, creating a larger sleeve. Furthermore, the two groups were not comparable, as the gastric bypass group had substantially less female patients and less total body fat. ${ }^{12}$

Even if it is proven that gastric bypass has better outcomes than sleeve gastrectomy on T2DM (beyond 5 years), it would save a substantial number of patients (50-80\%) from undergoing intestinal surgery, which has a plethora of serious complications, reserving failures to a conversion with a duodenal switch. It would also bring more patients to a cure worldwide overall, as sleeve is faster, easier and less complicated to perform than gastric bypass (at least double).

Finally, Cohen and colleagues have performed simple duodeno-jejunal bypass in patients with T2DM (as a duodenum bypassing intervention) and the results were inferior to those with sleeve gastrectomy alone. ${ }^{13}$ However, when sleeve gastrectomy was added to duodeno-jejunal bypass, $96 \%$ of remission was noted, rendering the intervention metabolically active. ${ }^{14}$ In conclusion, when one looks at a period of 10 years or more, the combination of sleeve gastrectomy first, followed by duodenal switch when indicated, will be superior for T2DM remission than a gastric bypass.

Department of Surgery, Hopital du Sacre Coeur, 315 Place D'Youville, Montreal, QC H2Y OA4, Canada. gagner.michel@gmail.com

\section{Competing interests}

The author declares associations with the following companies: Covidien, Ethicon, Gore, MID,
Transenterix. See the article online for full details of the relationships.

1. Cohen, R. Sleeve gastrectomy: the ideal option for metabolic surgery? Nat. Rev. Endocrinol. 9, doi:10.1038/nendo.2013.100-c1

2. Gagner, M. Obesity: Sleeve gastrectomy - the ideal choice for weight-loss surgery. Nat. Rev. Endocrinol. 9, 382-384 (2013).

3. Buchwald, H. et al. Bariatric surgery: a systematic review and meta-analysis. JAMA 292, 1724-1737 (2004).

4. Buchwald, H. et al. Weight and type 2 diabetes after bariatric surgery: systematic review and meta-analysis. Am J. Med. 122, 248.e5-256.e5 (2009).

5. Mingrone, G. et al. Bariatric surgery versus conventional medical therapy for type 2 diabetes. N. Engl. J. Med. 366, 1577-1585 (2012).

6. Garrido-Sanchez, $L$ et al. Bypass of the duodenum improves insulin resistance much more rapidly than sleeve gastrectomy. Surg. Obes. Relat. Dis 8, 145-150 (2012).

7. Chikunguwo, S. M. et al. Analysis of factors associated with durable remission of diabetes after Roux-en-Y gastric bypass. Surg. Obes. Relat. Dis. 6, 254-259 (2010).

8. Karamanakos, S. N., Vagenas K, Kalfarentzos, F. \& Alexandrides, T. K. Weight loss, appetite suppression, and changes in fasting and postprandial ghrelin and peptide-YY levels after Roux-en-Y gastric bypass and sleeve gastrectomy: a prospective, double blind study. Ann. Surg. 247, 401-407 (2008).

9. Kehagias, I., Karamanakos, S. N., Argentou, M. \& Kalfarentzos, F. Randomized clinical trial of laparoscopic Roux-en-Y gastric bypass versus laparoscopic sleeve gastrectomy for the management of patients with $\mathrm{BMl}<50 \mathrm{~kg} / \mathrm{m}_{2}$. Obes. Surg. 21, 1650-1656 (2011).

10. Lee, W. J. et al. Gastric bypass vs sleeve gastrectomy for type 2 diabetes mellitus: a randomized controlled trial. Arch Surg. 146 143-148 (2011).

11. Schauer, P. R. et al.Bariatric surgery versus intensive medical therapy in obese patients with diabetes. N. Engl. J. Med. 366, 1567-1576 (2012).

12. Kashyap, S. R. et al. Metabolic effects of bariatric surgery in patients with moderate obesity and type 2 diabetes: analysis of a randomized control trial comparing surgery with intensive medical treatment. Diabetes Care 36 2175-2182 (2013).

13. Klein, S. et al. Moderate effect of duodenaljejunal bypass surgery on glucose homeostasis in patients with type 2 diabetes. Obesity (Silver Spring) 20, 1266-1272 (2012).

14. Kasama, K. et al. Laparoscopic sleeve gastrectomy with duodenojejunal bypass: technique and preliminary results. Obes. Surg. 19, 1341-1345 (2009). 